\title{
BMJ Open Unmet needs in occupational health: prevention and management of viral hepatitis in healthcare workers in Ho Chi Minh City, Vietnam: a mixed- methods study
}

\author{
Tran Nguyen (D) , ${ }^{1}$ Trang Pham, ${ }^{2,3}$ Hong K Tang, ${ }^{4}$ Loc Phan, ${ }^{2}$ Gary Mize, ${ }^{2}$ \\ William M Lee, ${ }^{1,2}$ Robert Gish, ${ }^{2}$ Amy Trang, ${ }^{2}$ Anh Le, ${ }^{2}$ Hai T Phan, ${ }^{5}$ Binh T Nguyen, ${ }^{6}$ \\ Doan $\mathrm{Y} \mathrm{Dao}^{2,7}$
}

To cite: Nguyen T, Pham T, Tang HK, et al. Unmet needs in occupational health: prevention and management of viral hepatitis in healthcare workers in Ho Chi Minh City, Vietnam: a mixedmethods study. BMJ Open 2021;11:e052668. doi:10.1136/ bmjopen-2021-052668

- Prepublication history and additional supplemental material for this paper are available online. To view these files, please visit the journal online (http://dx.doi.org/10.1136/ bmjopen-2021-052668).

TN and TP contributed equally.

Received 21 April 2021 Accepted 27 September 2021

D) Check for updates

(c) Author(s) (or their employer(s)) 2021. Re-use permitted under CC BY-NC. No commercial re-use. See rights and permissions. Published by BMJ.

For numbered affiliations see end of article.

Correspondence to

Dr Doan Y Dao; ddoa1@jhmi.edu

\section{ABSTRACT}

Objectives Vietnam is an endemic area for hepatitis $B$ virus and hepatitis $\mathrm{C}$ virus infection (HBV-HCV), yet its largest city, Ho Chi Minh City (HCMC), has no comprehensive policy to educate, screen, treat and protect healthcare workers (HCWs) from viral hepatitis. We conducted a mixed-methods study to document HBV-HCV infection rates, risk factors, local barriers and opportunities for providing education, screening and medical care for HCWs.

Design This mixed-methods study involved an HBV and HCV serological evaluation, knowledge, attitude and practice survey about viral hepatitis and many in-depth interviews. Descriptive statistics and thematic content analysis using inductive and deductive approaches were used.

Setting HCMC, Vietnam.

Participants HCWs at risk of viral hepatitis exposure at three hospitals in HCMC.

Results Of the 210 invited HCWs, 203 were enrolled. Of the 203 HCWs enrolled, 20 were hepatitis B surface antigenpositive, 1 was anti-hepatitis $\mathrm{C}$ antibody (anti-HCV Ab)positive, 57 were anti-hepatitis $B$ core Ab-positive and 152 had adequate anti-hepatitis $B$ surface $\mathrm{Ab}$ (anti-HBs Ab) titre ( $\geq 10 \mathrm{IU} / \mathrm{mL}$ ). Only $50 \%$ of the infected HCWs reported always using gloves during a clinical activity involving handling of blood or bodily fluid. Approximately $50 \%$ of HCWs were still not vaccinated against $\mathrm{HBV}$ following 1 year of employment. In-depth interviews revealed two major concerns for most interviewees: the need for financial support for HBV-HCV screening and treatment in HCWs and the need for specific HBV-HCV guidelines to be independently developed.

Conclusions The high HBV infection rate in HCWs coupled with inadequate preventive occupational practices among the population in HCMC highlight the urgent needs to establish formal policy and rigorous education, screening, vaccination and treatment programmes to protect HCWs from HBV acquisition or to manage those living with chronic $\mathrm{HBV}$ in Vietnam.

\section{INTRODUCTION}

Globally, there are more than 2 million occupational exposures to sharp injuries in

\section{STRENGTHS AND LIMITATIONS OF THIS STUDY}

$\Rightarrow$ This is the first mixed-method study to investigate hepatitis B virus and hepatitis C virus infection (HBV$\mathrm{HCV}$ ) infection and risk factors among healthcare workers (HCWs); as well as local practice and barriers in HBV-HCV prevention among HCWs in $\mathrm{Ho}$ Chi Minh City, the largest city in Vietnam.

$\Rightarrow$ HCWs from national tertiary-level, city-level and district-level hospitals, which represent the three major healthcare system levels in Vietnam, were recruited, aiming to provide representative information regarding HBV-HCV for quantitative and qualitative data.

$\Rightarrow$ The in-depth interviews were conducted with both infected and non-infected HCWs from multiple professional and administrative levels among the participating hospitals to obtain diverse perspectives on local HBV-HCV practice and barriers.

$\Rightarrow$ Data from in-depth interviews were analysed using a thematic content analysis approach; thus, results were more descriptive than explanatory.

$\Rightarrow$ Data regarding HBV vaccine uptake among HCWs in this study were self-reported, which might be subject to recall bias.

the healthcare setting annually. ${ }^{1}$ The most common causes of postexposure infections are hepatitis B virus (HBV), hepatitis $\mathrm{C}$ virus (HCV) and HIV. ${ }^{1-3}$ Owing to the high prevalence of viral hepatitis infections in the general population in Vietnam-a low-income to middle-income country where an estimated $8.4 \%$ of the population are living with chronic HBV and another $1.1 \%$ of the population have chronic $\mathrm{HCV}^{4}{ }^{5}$-it is expected that Vietnamese healthcare workers (HCWs) are at greater risk for exposure and infection from these pathogens. 
Vietnamese HCWs are at risk of percutaneous needle stick injuries, especially in those with high frequency of contact with blood and bodily fluid, providing more opportunity for occupational exposure to HBV-HCV. ${ }^{6} 7$ The incidence rate of acquiring HBV infection after exposure was 25 times higher than that of acquiring HIV after exposure (50 cases per 100000 person-year vs 0.2 cases per 100000 person-year). ${ }^{8}$ In a study involving occupational exposure in HCWs at multiple hospitals in Ha Noi, Vietnam, Duong found that $64.8 \%$ of HCWs were exposed to sharp injuries at least once a year. This group of HCWs includes primarily nurses and physicians who worked directly with blood and bodily fluids or sharp instruments. ${ }^{8}$ In spite all of these statistics, Nguyen KTM and Nguyen revealed that $36.5 \%$ of nurses still did not have appropriate knowledge on prevention of occupational exposure to viral hepatitis and that about $10 \%$ of individuals did not follow the standard procedures for occupational exposure. ${ }^{9}$ Notably, most of the incidents were not reported to higher administrative levels. When these incidents occurred, they were not cared for in a timely and appropriate manner. ${ }^{7}$ Oftentimes, the sources of infection remained unknown. ${ }^{8}$

In Vietnam, viral hepatitis is a reportable infectious disease, but this only applied to hospitals that are dedicated to infectious disease specialty care and at the central government level. National recommendations for occupational exposure for prevention and management of infectious diseases, including viral hepatitis, have been issued but not mandated. According to the Infectious Disease Control and Prevention Act, viral hepatitis is in category $\mathrm{B}$, which is highly infectious and could lead to death. ${ }^{10}$ There is a lack of guidelines or step-by-step guidance for implementation or monitoring of viral hepatitis in healthcare settings. Moreover, funding to implement the national recommendations for infectious disease and viral hepatitis was not appropriated. As a result, procedures for employment screening and postexposure testing and management for viral hepatitis in HCWs were not uniformly or systematically implemented across healthcare settings in Vietnam. ${ }^{8}$ Instead, the procedures were only implemented at the individual healthcare centre's discretion. Furthermore, because of the lack of specific guidelines for viral hepatitis occupational health procedures, many hospitals in Vietnam adopted HIV guidelines instead. This approach resulted in low HBVHCV awareness, prevention and postexposure management in Vietnam. ${ }^{11}$

Pre-exposure vaccination for $\mathrm{HBV}$ has been highly successful in reducing HBV infection in HCWs. Rates of use in Vietnam are unknown, and no such intervention exists to prevent transmission. Similarly, hepatitis B immune globulin (HBIG) may be recommended as postexposure prophylaxis (PEP), but there are no formal recommendations available for PEP for HCWs exposed to HBV in Vietnam, nor is there data on availability of HBIG in these resource-limited and highly heterogeneous care settings. ${ }^{8}$ Thus, it is necessary to further understand

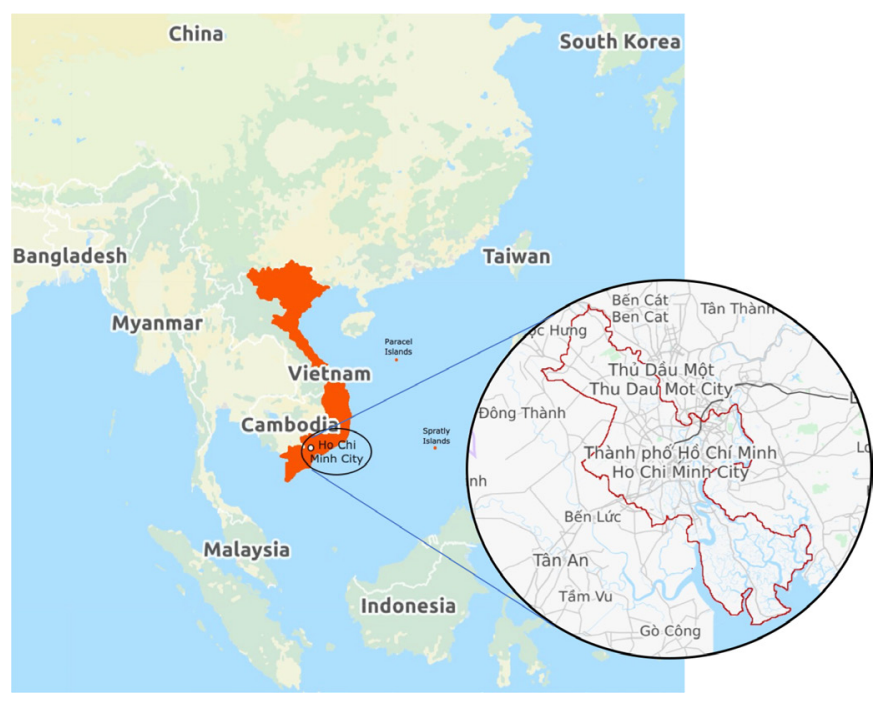

Figure 1 Vietnam, red S shape, is located in Southeast Asia. Ho Chi Minh City, enlarging circle, is located in southern Vietnam.

current practices with a mind toward the resource limitations of Vietnam and other developing regions.

In this study, we conducted a serosurvey of HBV-HCV; an assessment of viral hepatitis general knowledge, attitude and risk behaviours; and in-depth interviews in a cohort of HCWs in Ho Chi Minh City (HCMC). The in-depth interviews focused on Vietnam national circular, in-house protocol and procedures relating to occupational exposure for HBV-HCV prevention and management in HCWs. The study aimed to better understand the local needs and barriers for screening, prevention and linkage to care as well as best practices regarding occupational exposure to HBV-HCV in HCWs in HCMC.

\section{METHODS}

\section{Study setting}

The study was conducted in three hospitals in HCMC, Vietnam (figure 1). A low -to-middle income country, Vietnam is located in Southeast Asia and has a population of 97 million. With a population of 12 million, HCMC has an estimated prevalence of $7.8 \%$ for HBV and $2.2 \%$ for $\mathrm{HCV}$ in its community. ${ }^{12} 13$

The HCMC hospital system, with 91 public hospitals as of 2016, is divided into 3 levels: tertiary hospital (central government-level hospital), general hospital at city level, and general hospital at district level. ${ }^{14}$ In this study, we purposefully selected one hospital representing each of the hospital system levels to join the study. The study protocols were approved by institutional review Boards at Pham Ngoc Thach University of Medicine, a local medical school in HCMC, and at each of the participating hospitals.

\section{Study design and methods}

The study design comprised two parts: (1) an observational portion involving a Knowledge, Attitude and 
Practice (KAP) survey and serological screening for HBV-HCV and (2) in-depth interviews. For the former, a simple random sample of 210 participants, including 70 from each of the 3 hospitals representing 3 levels of hospital system in HCMC, were enrolled. The 210person sample was derived based on several factors: an estimate of $4000 \mathrm{HCWs}$ who worked at the 3 participating hospitals (unpublished data), a 0.05 margin of error at a $95 \%$ confidence level and the reported rate of infection of $15 \%$ for $\mathrm{HBV}$ and $2 \%-5 \%$ for $\mathrm{HCV}$ in HCWs in Vietnam. ${ }^{11}{ }^{15}$ To achieve the sample size of 210 and assume $70 \%$ response rate from invitees, each participating hospital selected 120 participants based on their staff directories and provided the study team the list of participants. Next, random selection of prospective participants from the lists was performed in Excel using the RAND function. Potential participants generated from this random selection process were invited to participate in the study. Participant recruitment took about 3 days to get 70 of 120 prospective participants.

The KAP questionnaire survey included demographics information (age, gender, educational level, type of clinical work, total years of clinical activity and income levels) and questions related to HBV-HCV knowledge, risk factors outside of the workplace, occupational exposures, HBV vaccination status and overall health status (online supplemental file 3). The questionnaires were initially developed based on the Behavioural Theory Framework and subsequently validated for Vietnamese in the USA and Vietnam. ${ }^{16}$

The in-depth interviews (ie, qualitative portion) were conducted within 2 weeks after the survey and screening. All participants were assigned a study ID. Participants who took the survey questionnaires and agreed to phlebotomy were invited to participate in the in-depth interviews. Those who agreed to in-depth interviews were stratified into seniority status, viral hepatitis infection status, and administrative role in the participating hospitals. Specifically, we applied a quota sampling approach to include participants with different levels of clinical experience ( $<5$ years vs $>5$ years), level of administrative responsibility (chief attending physician or chief nurse), viral hepatitis infection status (infected or naive) and professional levels (physicians, nurse/midwives, medical laboratory technician). In-depth interview was organised on a rolling basis, with each hospital having a maximum of 10 interviewees. We ended the interview at information saturation. This information saturation was at the sample size of 28 interviewees. In-depth interview was conducted by trained interviewers in Vietnamese. All interviewee information was deidentified. A semistructured questionnaire was used to guide the in-depth interview (online supplemental file 2).

\section{Participant recruitment and cascade of care follow-up}

To recruit participants into the serological screening and survey questionnaire portion, each of the three participating hospitals sent invitations internally to a maximum of 120 official full-time HCWs. We aimed to reach $210 \mathrm{HCWs}$ (expected response rate of approximately $70 \%$ ). To be included, HCWs needed to be 18 years or older and working in areas that required frequent contact with blood or bodily fluid. On completion of the screening tests and survey, a thank you gift card having the value of US $\$ 5$ was provided to participants. Within 2 weeks, results with written interpretation of serological testing and recommendations were returned to participants. Coupons offering free HBV vaccine were provided to $\mathrm{HBV}$-naive individuals (negative for hepatitis B surface antigen (HBsAg), antihepatitis B core antibody (antiHBcAb) and antihepatitis B surface (anti-HBs)) and free follow-up coupons were provided to individuals who were HBsAg-positive and/ or anti-HCV-positive. These follow-up coupons include free liver assessments (confirmatory HCV RNA, comprehensive metabolic panel and complete blood count) and free Fibroscan and hepatology consultation at an independent contracted medical centre. If treatment for HBV or HCV is indicated, the costs of treatment were reimbursed by national public health insurance. All the study participants had public health insurance coverage.

For the qualitative phase, participants were also invited to participate in a 1 hour, follow-up in-depth interview regarding barriers and facilitating factors in viral hepatitis prevention in the workplace and measurement of workplace occupational exposures. Twenty-eight participants were recruited, ${ }^{17} 18$ reaching data saturation. Trained interviewers used a semistructured questionnaire to collect data and provided interviewees US $\$ 5$ incentives after completing the session.

\section{Viral hepatitis serological testing}

Participants were screened for HBV and HCV. HBsAg was tested using a fully multivalent assay with high sensitivity in detecting HBV mutants to determine those who were positive for HBsAg. ELISA assay was performed following the manufacturer's instructions including serum anti-HBs and anti-HBcAb. HCV was screened with serum antihepatitis $\mathrm{C} \mathrm{Ab}$ (anti-HCV). All the screening tests for HBV-HCV were performed with Elecsys (Roche Diagnostics). Results were certified by a physician before being provided to screening participants.

\section{Data management and statistical analysis}

All surveys, interviews, transcriptions and coding of the qualitative data were done in Vietnamese. All surveys were checked for completeness. Missing items were not included in data analysis. Data were stored in Research Electronic Data Capture (REDCap). Demographic characteristics and risk factors for HBV-HCV and KAP data were reported as mean and SD for continuous variables and proportions for categorical variables, and subsequently compared between the groups with and without HBV or HCV. 
For survey questionnaires, KAP variables were coded as True (Applicable for) or False (Not Applicable for) for HBV, HCV or both HBV and HCV. Infection status was grouped as $\mathrm{HBsAg-positive} \mathrm{vs} \mathrm{HBsAg}$-negative for HBV and anti-HCV-positive vs anti-HCV-negative for HCV. Lab tests were merged with survey data, then cleaned and managed in STATA V.17. Data analysis was performed with univariate and bivariate statistics: the Cochran-Armitage trend test was used for continuous variables; the $\chi^{2}$ was used for categorical data. Significance level of 0.05 was used. All analyses used SAS V.9.4.

In-depth interviews were recorded and then transcribed into word documents, coded by two independent coders. Thematic content analysis using hybrid approach of inductive and deductive coding and theme development was performed in Excel. Initial codes were generated deductively and fitted into a preexisting coding framework based on the structure of the questionnaire and each label was defined based on the transcripts. We summarised the transcripts and outlined the key points addressed by the participants (which were prespecified before the interview or newly occurred in the conversation) to identify themes and patterns in the data. Themes were further clustered and assigned succinct phrases to describe the underpinning meanings.

\section{Patient and public involvement}

Patients or the public were not involved in this study.
RESULTS

\section{Sociodemographic characteristics of study participants}

There were 210 HCWs invited from 3 hospitals. Seven HCWs were non-clinical staff and excluded from the study. Of 210 invited HCWs, 203 (96.7\%) completed the demographics and KAP survey questionnaires and serological testing for HBV-HCV (table 1). Of the 203 HCWs, 39 were physicians, 140 were nurses and midwives, and 24 were technicians and nurse assistants. Overall, the age range was from 21 to 59 years old with a mean of 34.49. The majority of the 203 HCWs were female (83\%). Approximately $95 \%$ of the enrolled HCWs completed at least a technical or vocational degree, and more than half $(54.5 \%)$ worked in a clinical environment for less than 10 years. Among three groups of HCWs (physicians, nurses/ midwives and technicians/nurse assistants), most females (127 of 168) were nurses and midwives. All doctors graduated from university; and the majority of nurses, midwives, technicians and nurse assistants competed high school and vocational school.

\section{Serological characteristics of the study participant}

Twenty (9.8\%) of 203 HCWs were positive for HBsAg. Of 20, $17(85 \%)$ knew their viral hepatitis status; this included 4 doctors, 15 nurses and 1 technician. Nurses had similar rate of HBV infection at 10.7\% (15 of 140) compared with doctors at $10.2 \%$ (4 of 39). Technician and nurse assistant had the lowest rate of HBV infection with 1 infected person of 20 (5.0\%) HCWs. Four (1.97\%) were indeterminate with only positive anti-HBcAb and

Table 1 Baseline demographic characteristics of $203 \mathrm{HCWs}$

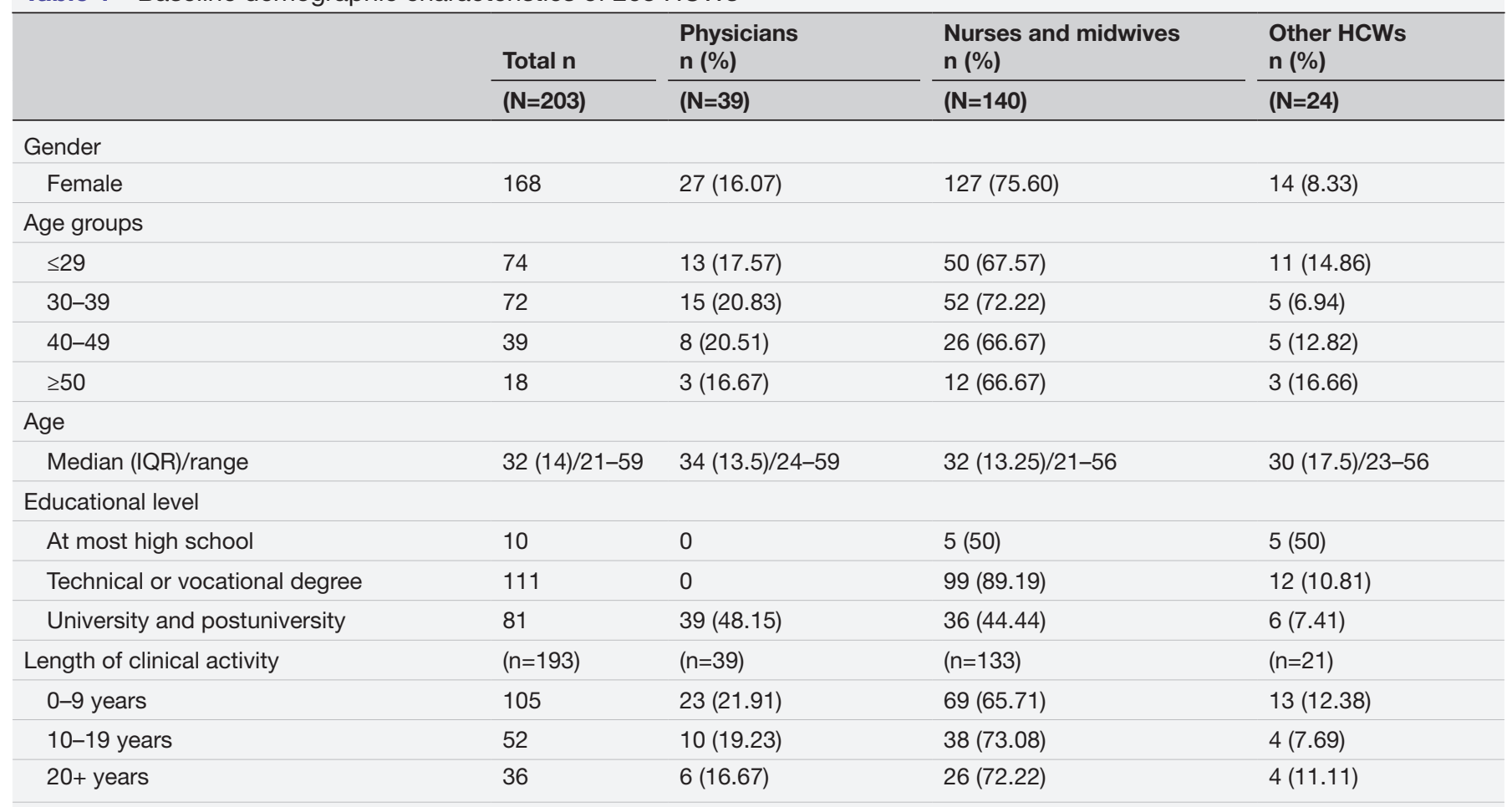

HCW, healthcare workers. 
Table 2 Demographic characteristics between HBsAg (+) and HBsAg (-) groups

\begin{tabular}{|c|c|c|c|c|}
\hline & Total & HBsAg (+) & HBsAg (-) & \\
\hline & $(n=203)$ & $(n=20)$ & $(n=183)$ & $P$ value \\
\hline Gender, n (\%) & & & & 0.731 \\
\hline \multicolumn{5}{|l|}{ Age } \\
\hline Median (IQR) & $32(14)$ & 35 (13.5) & $31(14)$ & \\
\hline Educational level, n (\%) & $(n=202)$ & $(n=20)$ & $(n=182)$ & 0.4188 \\
\hline High school or lower & $10(4.95)$ & 0 & $10(5.49)$ & \\
\hline Technical or vocational degree & $111(54.95)$ & $10(50)$ & $101(55.49)$ & \\
\hline University and postuniversity & $81(40.10)$ & $10(50)$ & $71(39.01)$ & \\
\hline Other HCWs & $20(10.05)$ & $1(5)$ & $19(10.61)$ & \\
\hline Length of clinical work, n (\%) & $(n=193)$ & $(n=19)$ & $(n=174)$ & 0.269 \\
\hline $0-9$ years & $105(54.40)$ & $7(36.84)$ & $98(56.32)$ & \\
\hline $10-19$ years & $52(26.94)$ & $7(36.84)$ & $45(25.86)$ & \\
\hline $20+$ years & $36(18.65)$ & $5(26.32)$ & $31(17.82)$ & \\
\hline
\end{tabular}

required follow-up testing. There were 27 (13.3\%) who were susceptible to HBV infection with negative HBsAg, anti-HBs and anti-HBc. Among those who were naive, there were 3 physicians $(7.7 \%, 3$ of 39$), 18$ nurses and midwives $(12.9 \%, 18$ of 140$)$ and 6 technicians $(25 \%, 6$ of 24). Ninety-nine $(48.77 \%)$ were immune from $\mathrm{HBV}$ vaccination with positive anti-HBs, and $53(26.11 \%)$ were with positive anti-HBs and anti-HBc. Among those who were vaccinated, there were 19 physicians $(58 \%, 19$ of 39), 69 nurses and midwives $(49 \%, 69$ of 140), and 11 technicians $(46 \%, 11$ of 24). Interestingly, 10 of these $99 \mathrm{HCWs}$ reported never receiving $\mathrm{HBV}$ vaccine. Regarding $\mathrm{HCV}$, there was only one person $(0.5 \%)$ who tested positive for anti-HCV and negative for HCV RNA. This person later reported already having HCV treatment 10 years prior.

\section{Comparison between HBV seropositive and HBV seronegative groups}

We divided the participants into two groups: 20 HCWs that were HBsAg-positive and 193 HCWs that were HBsAgnegative. As shown in table 2, there were no significant differences in demographic characteristics between the two groups. Both groups were approximately $80 \%$ female, and the age range was 25-54 years old and 21-59 years old. The majority of participants in both groups were nurses and midwives, the second most populous group was physicians. There was no difference in educational level or length of clinical work between the two groups. Regarding risk factors for HBV infection, a higher percentage of the HBV seropositive group had family members with HBV infection (60\% vs $15 \%, \mathrm{p}<0.0001)$ (table 3 ). Seventy per cent of the seronegative group reported no family member with either HBV or HCV, compared with $30 \%$ in the seropositive group. The seropositive group had a higher percentage of participants with daily exposure to blood and bodily fluid compared with the seronegative group (90\% vs 69\%). However, the difference was not significant $(p=0.054)$. There was no difference in the time since last check-up with HBV screening. However, rate of vaccine uptake was higher in the seronegative groups $(76 \%$ vs $30 \%, \mathrm{p}=0.0001)$. There were no differences in risks of hepatitis transmission, including prior blood transfusion, tattoo, illicit drug use or unprotected sex; except that 2 of the 20 the seropositive group (10\%) reported sharing needles in the past compared with none in the seronegative group $(\mathrm{p}<0.0001)$.

\section{Assessment of KAP}

According to the KAP survey (online supplemental table 1), the majority of HCWs provided correct answers to questions on modes of HBV-HCV transmission including sharing toothbrushes, sharing needles, sexual intercourse and during birth. However, 17\% (35 of 203) of HCWs believed that smoking could cause hepatitis, including 7 physicians, 23 nurses and midwives, and 5 other HCWs. Moreover, almost half (44\%, 90 of 203) thought that hepatitis could be spread by sharing utensils; this group included 19 physicians, 63 nurses and midwives, and 8 other HCWs. Twenty-nine per cent (58 of 203) also believed that sneezing could spread hepatitis, 
Table 3 Risk factors between HBsAg (+) and HBsAg (-) groups

\begin{tabular}{|c|c|c|c|c|}
\hline & \multirow{2}{*}{$\begin{array}{l}\text { Total } \\
(n=203)\end{array}$} & \multirow{2}{*}{$\begin{array}{l}\text { HBsAg (+) } \\
(n=20)\end{array}$} & \multirow{2}{*}{$\begin{array}{l}\text { HBsAg (-) } \\
(n=183)\end{array}$} & \multirow[b]{2}{*}{$P$ value } \\
\hline & & & & \\
\hline Frequency of exposure to blood and bodily fluids, $n(\%)$ & $(n=197)$ & $(n=20)$ & $(n=177)$ & 0.054 \\
\hline Every day & $141(71.57)$ & $18(90)$ & $123(69.49)$ & \\
\hline Not every day & $56(28.4)$ & $2(10)$ & $54(30.51)$ & \\
\hline Family member with viral hepatitis, $\mathrm{n}(\%)$ & $(n=203)$ & $(n=20)$ & $(n=183)$ & $<0.0001$ \\
\hline Only HBV & $39(19.21)$ & $12(60)$ & $27(14.75)$ & \\
\hline Only HCV & $3(1.48)$ & 0 & $3(1.64)$ & \\
\hline Both HBV and HCV & $6(2.96)$ & 0 & $6(3.28)$ & \\
\hline None & $135(66.50)$ & $6(30)$ & $129(70.49)$ & \\
\hline Don't know and did not answer & $20(9.85)$ & $2(10)$ & $18(9.84)$ & \\
\hline Family with HBV vaccination, n (\%) & $(n=185)$ & $(n=18)$ & $(n=167)$ & 0.297 \\
\hline Yes & $147(79.46)$ & $16(88.89)$ & $131(78.44)$ & \\
\hline Last time of health check-up with HBV screening, $n$ (\%) & $(n=201)$ & $(n=20)$ & $(n=181)$ & 0.750 \\
\hline Last 6 months & $106(52.74)$ & $10(50)$ & $96(53.04)$ & \\
\hline 6 months to 1 year & $30(14.93)$ & $3(15)$ & $27(14.92)$ & \\
\hline More than 1 year & $32(15.92)$ & $5(25)$ & $27(14.92)$ & \\
\hline Health check without HBV screening & $29(14.43)$ & $2(10)$ & 27 (14.92) & \\
\hline No health check-up & $4(1.99)$ & 0 & $4(2.21)$ & \\
\hline Health check-up with HBV screening paid by, n (\%) & $(n=166)$ & $(n=18)$ & $(n=148)$ & 0.130 \\
\hline Self & $33(19.88)$ & $6(33.33)$ & $27(18.24)$ & \\
\hline Employer & $133(80.12)$ & $12(66.67)$ & $121(81.76)$ & \\
\hline Any medical conditions, $\mathrm{n}(\%)$ & $(n=199)$ & $(n=)$ & $(n=179)$ & \\
\hline Yes & $30(15.08)$ & $6(30)$ & $24(13.41)$ & 0.0492 \\
\hline History of transfusion, n (\%) & $(n=199)$ & $(n=20)$ & $(n=179)$ & 0.8383 \\
\hline Yes & $12(6.03)$ & $1(5)$ & $11(6.15)$ & \\
\hline Having tattoo, n (\%) & $(n=199)$ & $(\mathrm{n}=20)$ & $(n=179)$ & 0.9133 \\
\hline Yes & $11(5.53)$ & 1 (5) & $10(5.59)$ & \\
\hline Use of addictive drugs, $\mathrm{n}(\%)$ & $(n=199)$ & $(n=20)$ & $(n=179)$ & 0.6347 \\
\hline Yes & $2(1.01)$ & 0 & $2(1.12)$ & \\
\hline Sharing needles, n (\%) & $(n=201)$ & $(n=20)$ & $(n=181)$ & $<0.0001$ \\
\hline Yes & $2(1)$ & $2(10)$ & 0 & \\
\hline Use of immunosuppressants or steroids, $n$ (\%) & $(n=201)$ & $(n=19)$ & $(n=182)$ & 0.5137 \\
\hline Yes & $2(1)$ & 0 & $2(1.10)$ & \\
\hline No & $189(94.03)$ & $19(100)$ & $170(93.41)$ & \\
\hline Not sure & $10(4.97)$ & 0 & $10(5.49)$ & \\
\hline Contact with sex workers, n (\%) & $(\mathrm{n}=202)$ & $(\mathrm{n}=20)$ & $(n=182)$ & \\
\hline Often & $1(0.5)$ & 0 & $1(0.55)$ & \\
\hline Sometimes & 0 & 0 & 0 & \\
\hline Never & $201(99.5)$ & $20(100)$ & $181(99.45)$ & \\
\hline In LGBT community, n (\%) & $(n=202)$ & $(n=20)$ & $(n=182)$ & \\
\hline Yes & $1(0.5)$ & 0 & $1(0.55)$ & \\
\hline Use of condoms, n (\%) & $(n=183)$ & $(n=18)$ & $(n=165)$ & 0.2172 \\
\hline Always & $34(18.58)$ & $2(11.11)$ & $32(19.39)$ & \\
\hline Sometimes & $42(22.95)$ & $7(38.89)$ & $35(21.21)$ & \\
\hline Never & $107(58.47)$ & $9(50)$ & 98 (59.39) & \\
\hline
\end{tabular}


Table 3 Continued

\begin{tabular}{|c|c|c|c|c|}
\hline & Total & HBsAg (+) & HBsAg (-) & \\
\hline & $(n=203)$ & $(n=20)$ & $(n=183)$ & $P$ value \\
\hline Partners were screened for HBV/HCV, n (\%) & $(n=191)$ & $(n=18)$ & $(n=173)$ & 0.1218 \\
\hline Received hepatitis B vaccination, n (\%) & $(n=200)$ & $(n=20)$ & $(n=180)$ & 0.0001 \\
\hline Yes & $142(71)$ & $6(30)$ & $136(75.56)$ & \\
\hline
\end{tabular}

Statisitically significant values are indicated in bold.

HBsAg, hepatitis B surface antigen; HBV, hepatitis B virus; HCV, hepatitis C virus; HCW, healthcare workers; LGBT, lesbian, gay, bisexual and transgender.

including 10 physicians, 41 nurses and midwives, and 7 other HCWs. Regarding knowledge on natural course of HBV-HCV, the majority believed that asymptomatic people can have chronic HBV or HCV infection (89\%) and that HBV-HCV are lifelong infections which can cause liver cancer $(95 \%)$ and can be lethal $(86 \%)$. However, 21\% (43 of 203) of HCWs believed that hepatitis is not treatable; this group included 4 physicians, 34 nurses and midwives, and 5 other HCWs. The majority (83\%, 169 of 203) thought that they do not need to avoid contact with people infected with HBV-HCV. Answers regarding the hepatitis $\mathrm{B}$ vaccine revealed that most HCWs (93\%, 189 of 203) believed that the HBV vaccine is effective, though 21\% (42 of 203) perceived that the HBV vaccine has harmful side effects. Overall, physicians exhibit better knowledge compared with the two other groups.

\section{In-depth interview results}

The in-depth interviews were conducted with 28 HCWs at 3 hospitals (table 4). The four main themes identified from the data were 'awareness of prevention and management policy and protocol for viral hepatitis in place,' the local 'postexposure management,' how 'HBV-HCV were screened and managed during annual health check,' and 'stigma, disclosure and support.'

Awareness of occupational exposure policy and/or protocol

All respondents were aware of the Ministry of Health's policy on prevention and control of occupational injuries in HCWs, and the local policy was similar to the national circular. Also, they stated that the major focus of postexposure incident reporting was HIV, so HBV-HCV pathogens were not included in checks for postexposure incidents (93\%, 26 of 28).

Table 4 In-depth interviews summary

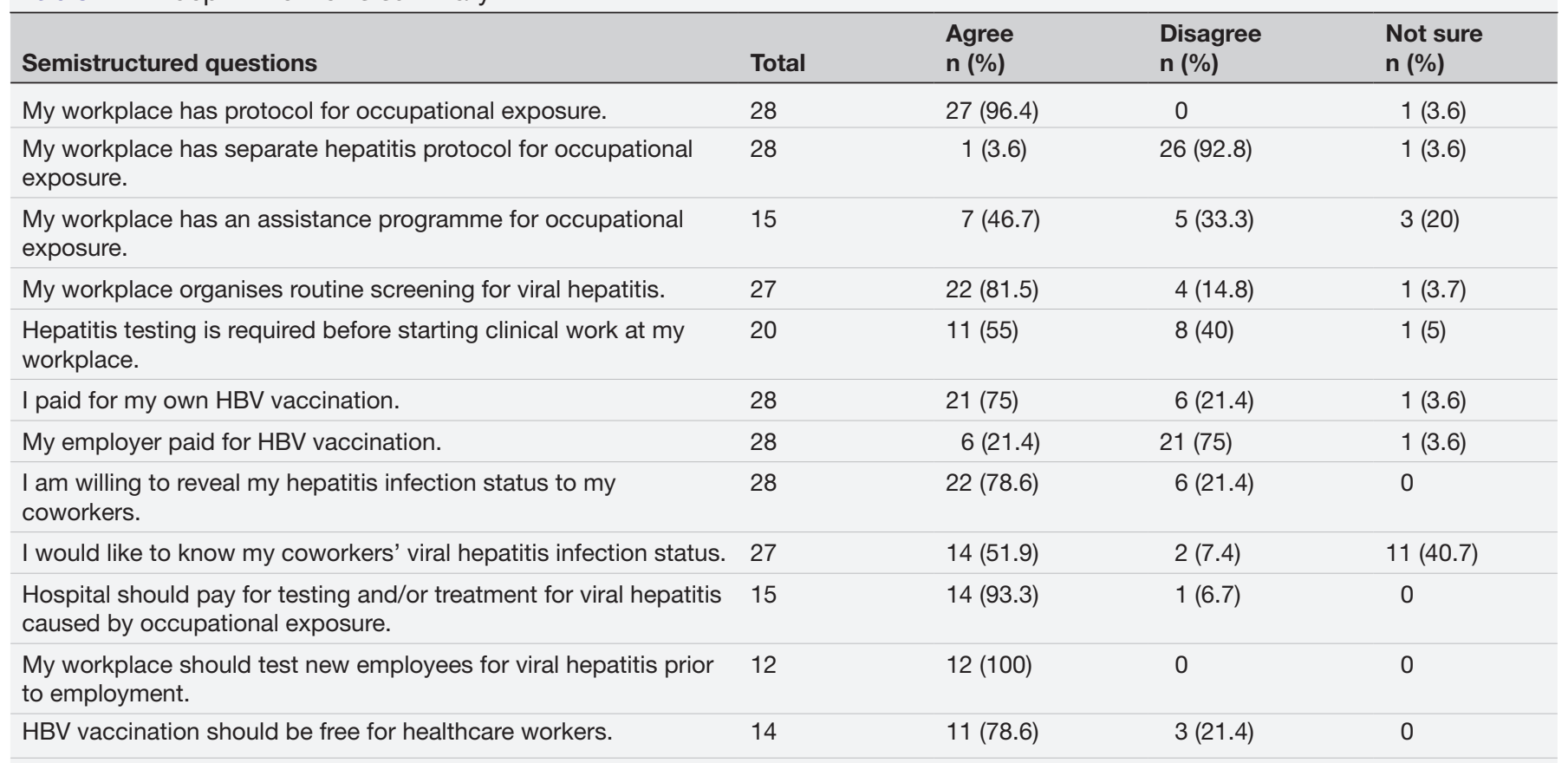

$\mathrm{HBV}$, hepatitis $\mathrm{B}$ virus. 
Quotes from in-depth interviews:

- 'The Ministry of Health did issue the guidelines for prevention of occupational exposure of needle sticks, so we applied it to our practice.'

- 'I don't think viral hepatitis is much different from HIV; that's why we can use the HIV protocol though.'

The national guidelines for prevention occupational exposure were more for needle sticks and HIV, but the HCWs applied it to viral hepatitis.

\section{Local occupational exposure management}

When asked about postexposure management, focusing on the local financial assistance programme for occupational exposure, $47 \%$ (7 of 15) reported receiving financial aid from the hospital for testing and medication for HIV exposure whereas 33\% (5 of 15) denied such support at their hospitals and had to self-pay the co-pay amount for examination and medication under their health insurance plan. Almost all interviewees (93\%, or 14 of 15) agreed that the hospital should pay for follow-up and/or treatment for hepatitis infection from occupational exposure, while 1 did not agree due to belief that hepatitis infection is not serious.

Most of the HCWs reported that they thought of HIV after exposure rather than HBV $(100 \%)$, and the postexposure reporting form did not ask whether the source of exposure was HBsAg or anti-HCV $(100 \%)$. All of the HCWs agreed that HBV and HCV should be mentioned in the postexposure reporting form and in the testing done after exposure for HCWs. Some HCWs said they had to pay for their HBV-HCV treatment because they did not want to use the national public health insurance's medications as it was not highly efficient, and demand the hospital to cover their treatment fee.

Quotes from in-depth interviews:

- 'I should think of HBV and HCV after being exposed to needle sticks, at that time, I reported only the HIV status of the patient.' 'Nothing in the accident reporting form related to HBV or HCV.'

- 'I just paid for my HBV treatment; I wanted to use better medication that were not in the public insurance's medication list.'

- 'I think it was OK for me to pay, but if the hospital can pay it, it would be a relief.'

\section{Screening and vaccination policy and the annual health check}

When asked about annual health check-ups for viral hepatitis, $48 \%$ (13 of 27) had only the HBV screening with HBsAg in their annual check-up organised and paid by their hospitals. Only 9 of 27 (33\%) had both HCV and HBV screening annually, which was paid by hospitals. Additionally, regarding testing requirements for new staff prior to start clinical work, $55 \%$ (11 of 20 ) received screening and vaccination recommendations during training or at the beginning of work, while $40 \%$ ( 8 of 20) reported that there was no such requirement. Before starting clinical work, about $55 \%$ (11 of 20) of interviewees reported that their hospitals required HBV and HCV tests, and 81.5\%
(22 of 27) of respondents stated that HBV and HCV were included in their annual health check.

Quotes from in-depth interviews:

- 'HBV and HCV were included in my health report when applying for a job in this hospital.'

- 'I got HBsAg and anti-HCV testing every year in the hospital health check day.'

If HBV vaccination is needed, $75 \%$ (21 of 28) HCWs paid for their own vaccination, and only $21.4 \%$ (6 of 28) confirmed they got free vaccination from their hospitals.

However, they agreed that:

- 'I got my vaccination during my medical training and I paid for it.'

- 'I got free vaccination at the hospital pharmacy department.'

- 'I think new employees should be tested for viral hepatitis before employment.'

- 'It would be the best if the screening and treatment fee can be covered by the hospitals.'

\section{Stigma, disclosure, and support}

Regarding 'stigma and support,' 79\% (22 of 28) of interviewees were willing to reveal their viral hepatitis status to coworkers whereas $21 \%$ (6 of 28) would like to keep it personal. Of those six interviewees, three interviewees voiced concern about stigma, and two reported that knowing their status would not change anything as they took measures to decrease transmission risk in the workplace. Alternatively, when asked if they would want to know their coworkers' viral hepatitis status, $52 \%$ (14 of 27 ) would like to know, $7 \%$ ( 2 of 27 ) would not like to know, and $41 \%$ (11 of 27 ) did not have strong opinions.

Quotes from in-depth interviews:

- 'I think it's OK to know other's status, so we can easily allocate the work and prevent spreading to the patient.'

Among those who would like to know, some voiced reasons including knowing risk of transmission with close contact, educating each other about preventive measures, and offering support to those with viral hepatitis infection. For those who would not want to know, they believed viral hepatitis status is private health information and should not be shared. Eleven interviewees reported that knowing coworkers' hepatitis status does not change their interactions. When asked if hepatitis infection could result in position reassignment, $36 \%$ (9 of 25) said no due to already high prevalance of viral hepatitis among HCWs, concern about discrimination and the fact that taking preventive measures is adequate to prevent tramission.

Furthermore, regarding HBV vaccination, $75 \%$ of interviewees (21 of 28) paid for their own vaccination, while 21\% (6 of 28) had cost covered by hospital. Most interviewees $(79 \%, 11$ of 14$)$ agreed that $\mathrm{HBV}$ vaccination should be free for all HCWs whereas 21\% (3 of 14) believed that vaccination should be self-paid due to financial constraint of the public health system and the affordability of vaccination when compared with HCWs' salaries. 


\section{DISCUSSION}

In this mixed-methods study, we documented the local best practices of occupational exposure and infection rates for HBV-HCV in HCWs in HCMC. Importantly, in-depth interviews revealed two major concerns for most interviewees. First, participants expressed the need for a specific guideline on HBV-HCV occupational exposure and prevention. This guideline should be independent from HIV guidelines. Second, policy on financial support for postexposure management for viral hepatitis in HCWs should be allocated.

In the observational portion, the study estimated a rate of HBsAg-positivity of $9.85 \%$ among HCWs working in HCMC. Compared with recent data on HBV prevalence of HCWs in other low-income to middle-income countries in Southeast Asia, HCWs in HCMC may have a higher rate of HBV than that of Thailand (5.3\%), Indonesia $(6.2 \%)$ and Laos (8\%). ${ }^{19-21}$ Regarding HCV, rate of anti-HCVpositive was much lower than HBV infection in this study $(0.5 \%$ vs $9.85 \%)$. Prior review also revealed lower average HCV prevalence of $1.6 \%$ in Southwest Asia, which ranges from $0.8 \%$ in Indonesia to $2.7 \%$ in Thailand. ${ }^{22}$ Although the most common scenario for both HBV and HCV exposure in HCWs is percutaneous injuries, HBV can survive outside the human body for at least 7 days and is many times more infectious than HCV or HIV. ${ }^{23-25}$ Moreover, $\mathrm{HBV}$ is the most easily transmitted bloodborne virus with a 6\%-30\% risk of infection from percutaneous exposure. Risk of acquiring HCV is lower, with a range from $2 \%$ to $4 \%{ }^{25}$

Although $71 \%$ of HCWs reported HBV immunisation, test results showed a low rate of vaccination (49\%) among three levels of HCWs with the uptake rate highest in physicians (58\%), followed by nurses $(49 \%)$ and technicians $(46 \%)$. The reported rate of vaccination is similar to a recent study done in Northern Vietnam $(68.8 \%)^{26}$ and other studies in South Africa (64.5\%). ${ }^{27} 28$ Low vaccine uptake may also be associated with HBV infection as demonstrated here and in previous studies. ${ }^{1929}$ There are several reasons to explain the low rate of vaccination.

First, the population of HCWs in our study did not generally get vaccination during early childhood. HBV vaccine, part of Vietnam's Expanded Programme on Immunisation, was first introduced in 1997 as a trial and was officially implemented in $70 \%$ of provinces of Vietnam only in $2004 .^{30}$ Therefore, national HBV vaccination for infants has only been active for 22 years. Since the average age of surveyed HCWs was 38 years old and the age range was from 25 to 54 years, the majority of HCWs was likely not vaccinated in their first year of life.

Second, most healthcare facilities in Vietnam do not require testing before starting work and vaccination against HBV, and do not incorporate viral hepatitis screening in annual check-up as demonstrated in the in-depth interviews. There were $10 \mathrm{HCWs}$ who reported never receiving $\mathrm{HBV}$ vaccine but they had lab results consistent with immunity from vaccination. On the other hand, there were six HCWs who reported previous vaccination but were HBsAg-positive. It is unclear if this is recall bias, that the initiation of vaccination was after $\mathrm{HBV}$ infection, or that the immunity from $\mathrm{HBV}$ vaccination had waned prior to HBV acquisition. The latter is less likely because $\mathrm{HBV}$ vaccine may confer protection from HBV infection for 30 years. ${ }^{31}$ Taken together, during employment process, it is important for viral hepatitis screening before starting work and that annual testing to avoid false assurance of vaccination in people who had acquired HBV infection prior to vaccine, especially in those who work in the healthcare settings with greater occupational risks. It is equally important to identify naive individuals for prompt vaccination to prevent $\mathrm{HBV}$ infection from occupational exposures.

Third, HBV vaccination was reported to be self-paid. Although several HCWs admitted the affordability of the $\mathrm{HBV}$ vaccine, they also mentioned free vaccination could encourage higher vaccine uptake. Besides financial barrier, other barriers, including unavailability of vaccine and busy work schedules, were also demonstrated in a prior study. ${ }^{32}$

We also identified high occupational risks: $71.5 \%$ of HCWs have daily exposure to blood and bodily fluid. Although almost all interviewees reported available protocols for occupational exposures, only one interviewee had a dedicated hepatitis protocol and the remaining interviewees followed HIV protocol. There was no available PEP for HBV exposure and no guidelines on follow-up testing and/or treatment. Most interviewees also voiced the need for an assistance programme for testing and/or treatment for hepatitis infection from occupational exposure. Therefore, there is a need for guidelines for occupational exposure of viral hepatitis and dedicated protocol for PEP, monitoring and treatment.

Similar to a recent study in Northern Vietnam, there was good overall knowledge of hepatitis transmission including parenteral, sexual and perinatal transmission. ${ }^{26}$ It seemed that the knowledge in these 203 HCWs in HCMC was better than that of previous studies conducted in Africa. ${ }^{29}{ }^{33}$ However, gaps of knowledge were identified in smoking, sharing foods and sneezing, which are not risk factors for hepatitis acquisition. Although there was no significant difference in knowledge score between the HBV-infected and non-infected groups, knowledge of hepatitis transmission is still important as HCWs are at a higher risk of contracting hepatitis via blood and bodily fluid exposure. However, a considerable proportion of HCWs did not believe viral hepatitis is treatable. This might be due to the lack of access to treatment knowledge as not everyone worked in the Hepatology department. From the in-depth interview, interviewees were aware of the inadequate knowledge of hepatitis and called for further education. Therefore, we suggest expanding annual training to include basic viral hepatitis core knowledge, testing and treatment as well as sequalae if unrecognised. As a result, this will facilitate vaccination uptake, awareness of modes of transmission and a proactive approach to follow up testing, especially after occupational exposure. 
This mixed-methods study reveals several gaps in hepatitis practice among HCWs in HCMC. First is the lack of pre-employment screening and routine surveillance for hepatitis. Second is inadequate guidelines for measures to be taken after hepatitis exposure. Therefore, we propose that hospitals should have mandatory pre-employment hepatitis screening for all prospective employees. This would help identify naive individuals who should be required to get HBV vaccination prior to starting their jobs to limit HBV infection from occupational exposures. This would also serve as an opportunity for those with hepatitis infection to know about their status. Additionally, for employees who will be at high risk of exposure to blood or body fluids on the job, postvaccination anti-HBs testing should be offered to identify individuals who did not achieve immunity with the standard HBV series. Those individuals who have documented prior $\mathrm{HBV}$ vaccination and negative antiHBsAb should receive a booster dose of $\mathrm{HBV}$ vaccine and be retested for immunity afterwards. We also propose that dedicated guidelines for HBV-HCV postexposure management will be available at the workplace for HCWs. Published guidelines should be at designated places, such as nursing stations or workrooms, for prompt access after occupational exposures. Following occupational exposure, skin sites that have been in contact with blood or bodily fluids should be washed with soap and water, and mucous membranes should be flushed with water. For HBV, prompt administration of HBIG or initiation of $\mathrm{HBV}$ vaccination should be initiated, depending on the HBV status of source patient and the exposed HCW. Appropriate HCWs should have follow-up serological testing (online supplemental table 2) ${ }^{34}$ For HCV, testing of source patient and exposed HCWs should be done as soon as possible. HCV PEP is not recommended. Schedules for follow-up serological testing after exposure for HCWs depends on HCV status of source patient and exposed HCW (online supplemental figure 1). ${ }^{35}$

Although this mixed-methods study was the first in Vietnam to provide more information about HBV-HCV in HCWs, there were several limitations. First, we do not intend to estimate the prevalence of HBV-HCV among HCWs in HCMC. Second, data regarding vaccine uptake was self-reported, which might be subject to recall bias. Also, there were no data regarding timing of vaccination in relation to timing of infection to determine vaccine efficacy. Despite these limitations, we still believe that this mixed-methods study offered insights into the needs for policy change to facilitate HBV vaccination, hepatitis surveillance, education and postexposure guideline changes. Furthermore, we propose effective interventions aimed at reduction of viral hepatitis disease burden in HCMC, Vietnam and would further support better analyses of anti-viral gaps and elimination targets that have been set for 2030 by WHO and Vietnam's National Action Plan for Viral Hepatitis Control and Prevention, period 2015-2019.

\section{CONCLUSION}

In conclusion, we documented that there are few guidelines for testing and treatment or best practices for occupational exposure to viral hepatitis in HCWs working in HCMC. Despite the high rate and risk of HBV infection in this population, only half of HCWs were vaccinated against HBV. A knowledge gap was also identified with the KAP survey that continuous medical education is crucial to improve the knowledge and to protect HCWs. This study is a call for an effort to enforce mandatory preemployment testing, routine surveillance, HBV vaccination and dedicated HBV-HCV postexposure guidelines and treatment for HCWs.

\section{Author affiliations}

${ }^{1}$ Department of Internal Medicine, The University of Texas Southwestern Medical Center, Dallas, Texas, USA

${ }^{2}$ Vietnam Viral Hepatitis Alliance, Reston, Virginia, USA

${ }^{3}$ University of Illinois at Chicago, Chicago, Illinois, USA

${ }^{4}$ Department of Epidemiology, Faculty of Public Health, Pham Ngoc Thach University of Medicine, Ho Chi Minh, Vietnam

${ }^{5}$ Medic Medical Center, Ho Chi Minh Clty, Vietnam

${ }^{6} \mathrm{Ho}$ Chi Minh City Department of Health, Ho Chi Minh City, Vietnam

${ }^{7}$ Center of Excellence for Liver Disease in Vietnam, Johns Hopkins University School of Medicine, Baltimore, Maryland, USA

\section{Twitter Robert Gish @robertgish}

Acknowledgements We thank Hung Vuong hospital, Nguyen Tri Phuong hospital and District five hospital in Ho Chi Minh City, Vietnam, for their support with recruitment and day-to-day study. We thank Abbott Vietnam, Roche Vietnam and Phuoc Thien Pharma for in-kind donation of test kits and vaccines. We thank all healthcare workers who participated in this study. We thank Kelly Schrank for her editorial services in preparing the manuscript for publication.

Contributors TP, HKT, AT, AL and DYD contributed to study design. TP, LP, AT, AL and HKT contributed to data collection. TN, TP and DYD contributed to data analysis and manuscript preparation. DYD, GM, RG, WML, HTP, BTN and HKT contributed to funding acquisition. DD is fully responsible for the overall content for the work and the conduct of the study, had access to the data, and controlled the decision to publish. All authors reviewed the manuscript and approved the submitted final version.

Funding The research was funded by AbbVie Inc. (grant no: 1450/QD-UBND).

Map disclaimer The depiction of boundaries on this map does not imply the expression of any opinion whatsoever on the part of BMJ (or any member of its group) concerning the legal status of any country, territory, jurisdiction or area or of its authorities. This map is provided without any warranty of any kind, either express or implied.

Competing interests None declared.

Patient consent for publication Not applicable.

Ethics approval Ethical approval was obtained by the Institutional Review Board of Pham Ngoc Thach University of Medicine (IRB no: 206a/DHYPNT-NCKH) and the Ho Chi Minh City Department of Health (no: 1397/SYT-NVY). All participants provided informed consent.

Provenance and peer review Not commissioned; externally peer reviewed.

Data availability statement Data are available on reasonable request. All data relevant to the study are included in the article or uploaded as online supplemental information. Deidentified data are stored in internal database and are available upon request to the corresponding author. All data relevant to the study are included in the article or uploaded as supplementary material.

Supplemental material This content has been supplied by the author(s). It has not been vetted by BMJ Publishing Group Limited (BMJ) and may not have been peer-reviewed. Any opinions or recommendations discussed are solely those of the author(s) and are not endorsed by BMJ. BMJ disclaims all liability and responsibility arising from any reliance placed on the content. Where the content includes any translated material, BMJ does not warrant the accuracy and reliability 
of the translations (including but not limited to local regulations, clinical guidelines, terminology, drug names and drug dosages), and is not responsible for any error and/or omissions arising from translation and adaptation or otherwise.

Open access This is an open access article distributed in accordance with the Creative Commons Attribution Non Commercial (CC BY-NC 4.0) license, which permits others to distribute, remix, adapt, build upon this work non-commercially, and license their derivative works on different terms, provided the original work is properly cited, appropriate credit is given, any changes made indicated, and the use is non-commercial. See: http://creativecommons.org/licenses/by-nc/4.0/.

\section{ORCID iD}

Tran Nguyen http://orcid.org/0000-0003-2611-317X

\section{REFERENCES}

1 World Health Organization. Protecting health-care workers preventing needlestick injuries, 2019. Available: https://www.who.int/ occupational_health/topics/needinjuries/en/ [Accessed 1 Apr 2021].

2 Beltrami EM, Williams IT, Shapiro CN, et al. Risk and management of blood-borne infections in health care workers. Clin Microbiol Rev 2000;13:385-407.

3 Prüss-Ustün A, Rapiti E, Hutin Y. Estimation of the global burden of disease attributable to contaminated sharps injuries among healthcare workers. Am J Ind Med 2005;48:482-90.

4 Nguyen VTT, Anh NT, Kato M. Estimates and projection of disease burden and economic analysis for hepatitis B in Viet Nam. J Viral Hepat 2018;25.

5 Nguyen VTT, Anh NT, Kato M. Estimates and projection of disease burden and investment case for hepatitis $C$ in Viet Nam. $J$ Viral Hepat 2018;25.

6 Ministry of Health. A model for preventing occupational viral hepatitis, 2009. Available: https://www.moh.gov.vn/web/phongchong-benh-nghe-nghiep/tin-van-ban-phap-quy/-/asset_publisher/ FAWwP4KSwRij/content/mo-hinh-phong-chong-viem-gan-virutnghe-nghiep [Accessed 1 Apr 2021].

7 Truong LT. Examining severity of, pathogen of, and management for occupational exposure incidents in healthcare providers at Quang Nam General Hospital from 2006-2011, 2011. Available: http://www. hics.org.vn/sites/default/files/attachment/123_truong_thi_ngoc_lan._ tim_hieu_muc_do_nguyen_nhan_va_cach_xu_tri_tai_nan_nghe_ nghiep_tai_bvdk_quang_nam.pdf [Accessed 1 Apr 2021].

8 Duong V. Examine occupational injuries in healthcare workers and intervention implementation in selected hospitals in HA Nol area. National library of Vietnam. No LA13.0636.3.2013, 2013. Available: http://luanan.nlv.gov.vn/luanan?a=d\&d=TTcFqWrIEluO2013.1.1\&e=vi-20-1-img-txIN- [Accessed 1 Apr 2021].

9 Nguyen KTM NH, Nguyen BN. Knowledge and practice in preventing occupational HBV exposure in nurses of Nguyen Dinh Chieu hospital in 2018, 2019. Available: https://www.researchgate.net/publication/ 333844383_KIEN_THUC_THUC_HANH_PHONG_BENH_VIEM_GAN B_NGHE_NGHIEP_CUA_DIEU_DUONG_LAM_SANG_BENH_VIEN_ NGUYEN_DINH_CHIEU_BEN_TRE_NAM_2018 [Accessed 1 Apr 2021].

10 Ministry of Health. Infectious disease prevention and control act. No 03/2007/QH12, 2007. Available: http://vbpl.vn/boyte/Pages/vbpqtoanvan.aspx? ItemID=12900 [Accessed 1 Apr 2021].

11 Ministry of Health. National action plan for prevention and control of viral hepatitis from 2015 to 2019, no 739/QD-BYT, 2015.

$12 \mathrm{Ngoc}$, Phan L, Ngo D. The burden of and barriers to care for hepatitis $\mathrm{C}$ virus (HCV) in Ho Chi Minh City, Vietnam: a comprehensive population-based prevalence study. Hepatology 2020;72.

13 Ngoc T, Phan L, Ngo D. The prevalence and characteristics of patients with hepatitis B virus (HBV) in Ho Chi Minh City (HCMC), Vietnam: implications for HBV elimination by 2030 . Hepatology 2020;72.

14 Ho Chi Minh department of health. RANK list of all public hospitals in Ho Chi Minh City, 2016. Available: http://www.medinet.hochiminhcity. gov.vn/thong-tin-khen-thuong-bo-nhiem/danh-sach-xep-hang- cac-co-so-y-te-cong-lap-tren-dia-ban-thanh-pho-ho-chi-minhcmobile1298-1205.aspx [Accessed 30 Jul 2021].

15 TQ D, Tran HM. Prevalence of HBV in healthcare professionals in Quang Binh hospital in 2012. J Prev Med Vietnam 2013;6:23:50.

16 Maxwell AE, Bastani R, Glenn BA, et al. Developing theoretically based and culturally appropriate interventions to promote hepatitis B testing in 4 Asian American populations, 2006-2011. Prev Chronic Dis 2014;11:E72.

17 Ashley K, Hagaman AW. How many interviews are enough to identify metathemes in multisited and cross-cultural research? Another perspective on Guest, Bunce, and Johnson's (2006) Landmark Study. Field Methods 2017;29:23-41.

18 Vasileiou K, Barnett J, Thorpe S, et al. Characterising and justifying sample size sufficiency in Interview-Based studies: systematic analysis of qualitative health research over a 15-year period. BMC Med Res Methodol 2018;18:148.

19 Chiarakul S, Eunumjitkul K, Vuttiopas S, et al. Seroprevalence and risk factors of hepatitis $B$ virus infection among health care workers at the Institute of Neurology. J Med Assoc Thai 2007;90:1536-45.

20 Wijayadi T, Sjahril R, le SI. Seroepidemiology of HBV infection among health-care workers in South Sulawesi, Indonesia. BMC Infect Dis 2018;18:279.

21 Black AP, Vilivong K, Nouanthong $P$, et al. Serosurveillance of vaccine preventable diseases and hepatitis $\mathrm{C}$ in healthcare workers from Lao PDR. PLoS One 2015;10:e0123647.

22 Petruzziello A, Marigliano S, Loquercio G, et al. Global epidemiology of hepatitis $C$ virus infection: an up-date of the distribution and circulation of hepatitis C virus genotypes. World J Gastroenterol 2016;22:7824-40.

23 Ciorlia LAS, Zanetta DMT. Hepatitis B in healthcare workers: prevalence, vaccination and relation to occupational factors. Braz J Infect Dis 2005;9:384-9.

24 Egro FM, Nwaiwu CA, Smith S, et al. Seroconversion rates among health care workers exposed to hepatitis $\mathrm{C}$ virus-contaminated body fluids: the University of Pittsburgh 13-year experience. Am J Infect Control 2017;45:1001-5.

25 Centers for Disease Control and Prevention. Sharps injuries: bloodborne pathogens, 2019. Available: https://www.cdc.gov/nora/ councils/hcsa/stopsticks/bloodborne.html [Accessed 10 Mar 2021].

26 Hang Pham TT, Le TX, Nguyen DT, et al. Knowledge, attitudes and medical practice regarding hepatitis $B$ prevention and management among healthcare workers in northern Vietnam. PLoS One 2019;14:e0223733.

27 Aaron D, Nagu TJ, Rwegasha J, et al. Hepatitis B vaccination coverage among healthcare workers at national hospital in Tanzania: how much, who and why? BMC Infect Dis 2017;17:786.

28 Ogoina D, Pondei K, Adetunji B, et al. Prevalence of hepatitis B vaccination among health care workers in Nigeria in 2011-12. Int J Occup Environ Med 2014;5:51-6.

29 Shao ER, Mboya IB, Gunda DW, et al. Seroprevalence of hepatitis B virus infection and associated factors among healthcare workers in northern Tanzania. BMC Infect Dis 2018;18:474.

30 Mohamed R, Desmond P, Suh D-J, et al. Practical difficulties in the management of hepatitis B in the Asia-Pacific region. J Gastroenterol Hepatol 2004;19:958-69.

31 Bruce MG, Bruden D, Hurlburt D, et al. Antibody levels and protection after hepatitis $B$ vaccine: results of a 30-year follow-up study and response to a booster dose. J Infect Dis 2016;214:16-22.

32 Auta A, Adewuyi EO, Kureh GT, et al. Hepatitis B vaccination coverage among health-care workers in Africa: a systematic review and meta-analysis. Vaccine 2018;36:4851-60.

33 Qin Y-L, Li B, Zhou Y-S, et al. Prevalence and associated knowledge of hepatitis B infection among healthcare workers in Freetown, Sierra Leone. BMC Infect Dis 2018;18:315.

34 Schillie S, Murphy TV, Sawyer M. Cdc guidance for evaluating healthcare personnel for hepatitis B virus protection and for administering postexposure management, 2013. Available: https://www.cdc.gov/ $\mathrm{mmwr} /$ preview/mmwrhtml/rr6210a1.htm [Accessed 1 Apr 2021].

35 Moorman AC, de Perio MA, Goldschmidt R. Testing and clinical management of health care personnel potentially exposed to hepatitis C virus - CDC Guidance, United States, 2020. Available: https://www.cdc.gov/mmwr/volumes/69/rr/rr6906a1.htm?s_cid= rr6906a1_w [Accessed 1 Apr 2021]. 